\title{
Joint Power Control and Blind Beamforming over Wireless Networks: A Cross Layer Approach
}

\author{
Zhu Han \\ Department of Electrical and Computer Engineering and Institute for Systems Research, University of Maryland, \\ College Park, MD 20742, USA \\ Email: hanzhu@glue.umd.edu \\ Farrokh R. Farrokhi \\ Department of Electrical and Computer Engineering and Institute for Systems Research, University of Maryland, \\ College Park, MD 20742, USA \\ Email: frfarrokhi@yahoo.com

\section{K. J. Ray Liu} \\ Department of Electrical and Computer Engineering and Institute for Systems Research, University of Maryland, \\ College Park, MD 20742, USA \\ Email:kjrliu@umd.edu
}

Received 6 January 2003; Revised 9 September 2003

\begin{abstract}
Traditional joint power control and beamforming achieve the targeted signal-to-interference-noise ratio (SINR) at the receivers by assuming the knowledge of the measurements of channel parameters and SINR. Blind beamforming is an effective technique for beamforming and channel estimation without the need of training sequences, thus not consuming extra bandwidth. In this paper, we propose a novel joint power control and blind beamforming algorithm that reformulates the power control problem in such a way that it does not need any prior knowledge and additional measurements in the physical layer. In contrast to the traditional schemes that optimize SINR and, as a result, minimize bit error rate (BER), our proposed algorithm achieves the desired BER by adjusting a quantity available from blind beamforming. By sending this quantity to the transmitter through a feedback channel, the transmit power is iteratively updated in a distributed manner in the wireless networks with cochannel interferences (CCIS). Our proposed algorithm is more robust to estimation errors. We have shown in both analysis and simulation that our algorithm converges to the desired solution. In addition, a Cramer-Rao lower bound (CRB) is derived to compare with the performance of our proposed joint power control and blind beamforming system.
\end{abstract}

Keywords and phrases: array signal processing, power control, distributed control, direction of arrival estimation.

\section{INTRODUCTION}

Over the past few decades, wireless communications and networking have witnessed an unprecedented growth, and have become pervasive much sooner than anyone could have imagined. One of the major challenges for the system design is the limited available radio frequency spectrum. Channel reuse is a common method to increase the wireless system capacity by reusing the same channel beyond some distance. However this introduces cochannel interference (CCI) that degrades the link quality. Two promising approaches to combat CCI are power control and antenna array processing. Power control is one direct approach toward minimizing CCI. The transmit powers are constantly adjusted. They are increased if the signal-to-interference-noise-ratios
(SINRs) at the receivers are low and are decreased if the SINRs are high. Such a process improves the quality of weak links and reduces the unnecessary transmit powers. Antenna array processing techniques such as beamforming can be applied to receive and transmit multiple signals that are separated in space. Hence, multiple cochannel users can be supported in each cell to increase the capacity by exploring the space diversity.

Many works have been reported in the literature for employing power control and beamforming to reduce CCI. Traditional beamformers such as minimum mean square error (MMSE) and minimum variance distortion response (MVDR) methods have been commonly employed [1]. In $[2,3]$, general frameworks for power control are constructed. Beamforming is a physical layer technique that can greatly 
increase receivers' SINR by using the signal processing algorithms, while power control is a media access control layer technique that can effectively control users' transmit powers to share the channels. Many joint power control and beamforming algorithms are proposed in $[4,5$, $6,7,8]$. Most of the existing works assume the availability of prior channel information and measurement of SINR.

As a majority of communication systems often struggle with the limited bandwidth constraint, it is desirable for the receiver with multiple antennas to steer to the desired direction and to estimate the transmit signals without consuming much channel bandwidth. By eliminating the training sequence overhead, used for estimation, and maximizing the channel capacity for information transmission, blind estimation and beamforming $[9,10,11,12,13,14,15,16]$ offer a bandwidth efficient solution to signal separation and estimation. Its importance also lies in the practical need for some communication receivers to equalize unknown channels without the assistance and the expense of training sequences.

Current methods of joint power control and beamforming $[4,5,6,7,8]$ assume perfect measurement of channel parameters and SINR at the receivers, which is very difficult to obtain in practice. Blind beamforming can estimate and separate, without the use of training sequences, the transmitted signals that suffer from the channel distortion and additive noise. The difficulties for joint power control and blind beamforming are to formulate such a cross-layer problem into a joint optimization problem, and develop an algorithm that can be self-trained and adaptively adjust the system parameters. In this paper, we present a novel joint power control and blind beamforming algorithm for a multicell multiantenna system. Based on a reformulated joint problem, our proposed algorithm optimizes the bit error rate (BER) using a quantity directly available from the blind beamforming and estimation, which avoids additional measurements mentioned above. Mobiles' transmit powers are updated in a distributed manner such that the CCI is effectively reduced. Convergence properties of the proposed algorithm are discussed. A Cramer-Rao lower bound (CRB) is derived to show the effect of power control on the symbol estimation performance in the networks. Simulation results illustrate that our algorithm converges to the desired solution and is more robust to channel estimation error compared with traditional joint power control and training-based beamforming algorithm.

The organization of this paper is as follows. In Section 2, we present the system model and the traditional joint power control and beamforming problem. In Section 3, first we choose a blind beamforming algorithm. Then we give the reformulated joint power control and blind beamforming problem. An adaptive algorithm is developed and a system is constructed. In Section 4, the convergence and uniqueness of the solution are analyzed. The CRB is derived to compare the performance. In Section 5, we evaluate our algorithm via numerical studies. In Section 6, we give the summary and conclusion.

\section{SYSTEM MODEL, BEAMFORMING AND POWER CONTROL}

Consider $K$ distinct cells in wireless networks where cochannel links exist. Each cell consists of one base station and its assigned $D$ mobiles. Antenna arrays with $M$ elements are used only at the base station and $M \geq D$. We assume that coherent detection is possible so that it is sufficient to model this multiuser system by an equivalent baseband model. Each link is affected by the slow Rayleigh fading. The propagation delay is far less than one symbol period. For uplink case, the ith base station antenna array's output vector is given by

$$
\begin{aligned}
& \mathbf{x}_{i}(t) \\
& =\sum_{k=1}^{K} \sum_{d=1}^{D} \sqrt{G_{k i}^{d} P_{k}^{d}} \alpha_{k i}^{d} \mathbf{a}_{k i}^{d}\left(\theta_{k i}^{d}\right) \cdot g_{k}^{d}\left(t-\tau_{k i}\right) s_{k}^{d}\left(t-\tau_{k i}\right)+\mathbf{n}_{i}(t),
\end{aligned}
$$

where $G_{k i}^{d}$ is path loss, $\alpha_{k i}^{d}$ is fading coefficient, $P_{k}^{d}$ is transmit power, $\mathbf{a}_{k i}^{d}\left(\theta_{k i}^{d}\right)$ is the $i$ th base station array response vector to the signal from the $d$ th mobile in the $k$ th cell at direction $\theta_{k i}^{d}, g_{k}^{d}(t)$ is shaping function, $s_{k}^{d}(t)$ is message symbol, $\tau_{k i}$ is the delay, and $\mathbf{n}_{i}(t)$ is thermal noise vector. We assume the synchronous transmission for all the users within the same cell, that is, $\tau_{i i}=0$, for all $i$. The synchronous assumption is reasonable because the symbol timing can be effectively controlled within each cell. We assume that the CCI from other cells is asynchronous for the desired signals within the cell and $\tau_{k i}, k \neq i$, is uniformly distributed within the symbol duration. We assume that the channels are flat fading and stable within a frame of hundreds of symbols. Define the impulse response from the $d$ th mobile in the $k$ th cell to the $p$ th element of the $i$ th base station as $h_{k i}^{d p}=\alpha_{k i}^{d} a_{k i}^{d p}\left(\theta_{k i}^{d}\right) r_{k i}^{d p}$, where $r_{k i}^{d p}$ includes the effect of the transmitter, receiver filter, and shaping function $g_{k}^{d}\left(t-\tau_{k i}\right)$. In the vector form, it is given by $\mathbf{h}_{k i}^{d}=\left[h_{k i}^{1 d}, \ldots, h_{k i}^{M d}\right]^{T}$. The sampled received vector for this $D K$ users and $M K$ antenna outputs multicell system at time $n$ is given by

$$
\mathbf{X}(n)=\mathbf{A} \mathbf{S}(n)+\mathbf{n}(n)
$$

where $\mathbf{X}(n)=\left[\mathbf{x}_{1}^{T}(n), \mathbf{x}_{2}^{T}(n), \ldots, \mathbf{x}_{K}^{T}(n)\right]^{T}, \mathbf{S}(n)=\left[\mathbf{S}_{1}^{T}(n)\right.$, $\left.\mathbf{S}_{2}^{T}(n), \ldots, \mathbf{S}_{K}^{T}(n)\right]^{T}, \mathbf{S}_{i}(n)=\left[s_{i}^{1}(n), \ldots, s_{i}^{D}(n)\right]^{T}, \mathbf{n}(n)$ is the sampled thermal noise vector, and

$$
\mathbf{A}=\left[\begin{array}{cccc}
\mathbf{A}_{11} & \mathbf{A}_{21} & \cdots & \mathbf{A}_{K 1} \\
\mathbf{A}_{12} & \mathbf{A}_{22} & \cdots & \mathbf{A}_{K 2} \\
\vdots & \vdots & \vdots & \vdots \\
\mathbf{A}_{1 K} & \mathbf{A}_{2 K} & \cdots & \mathbf{A}_{K K}
\end{array}\right]_{M K \times D K}
$$

where $\mathbf{A}_{i j}=\left[\sqrt{P_{i}^{1} G_{i j}^{1}} \mathbf{h}_{i j}^{1} \cdots \sqrt{P_{i}^{D} G_{i j}^{D}} \mathbf{h}_{i j}^{D}\right]$.

Let $\mathbf{w}_{i}^{d}$ be the beamforming weight vector for the $d$ th mobile in the $i$ th cell. Without loss of generality, we normalize the beamformer weight vector $\left\|\left(\mathbf{w}_{i}^{d}\right)^{H} \mathbf{h}_{i i}^{d}\right\|^{2}=1$, which will not change the receivers' SINRs. We assume that the transmitted signals from different sources are uncorrelated and 
zero mean, and the additive noise is spatially and temporally white with variance $\mathbf{N}_{i}=\sigma^{2} \mathbf{I}_{M \times M}$, where $\sigma^{2}$ is the thermal noise variance. The $d$ th user's SINR at its associated $i$ th base station's beamformer output is

$$
\Gamma_{i}^{d}=\frac{P_{i}^{d} G_{i i}^{d}}{\sum \sum_{(k, j) \neq(i, d)} P_{k}^{j} G_{k i}^{j}\left\|\left(\mathbf{w}_{i}^{d}\right)^{H} \mathbf{h}_{k i}^{j}\right\|^{2}+\left(\mathbf{w}_{i}^{d}\right)^{H} \mathbf{N}_{i} \mathbf{w}_{i}^{d}} .
$$

The issue in question here is how to find the users' beamforming vectors and transmit powers such that each user has the desired link quality and does not introduce unnecessary CCI to other users. In the rest of this section, we will briefly illustrate the traditional joint power control and beamforming.

An adaptive antenna array is designed to receive the signals from the desired directions and attenuate signals' radiations from other directions of no interest. The outputs of the array elements are weighted by a beamformer. In order to suppress the interferences, the beamformer places its nulls in the directions of interference sources and steers to the direction of the target signal. Some most popular beamformers are MMSE and MVDR beamformers [1]. In this paper, we will compare joint power control and MVDR beamforming method with our proposed blind scheme because MVDR beamformer is commonly used in the literature [4].

If the channel responses $\mathbf{h}_{i i}^{d}$ can be estimated, the beamforming vector can be calculated by the MVDR method, which minimizes the total interferences at the output of a beamformer, while the gain for the desired $d$ th user in the $i$ th cell is kept as a constant. The MVDR problem can be defined as

$$
\min _{\mathbf{w}_{i}^{d}}\left\|\left(\mathbf{w}_{i}^{d}\right)^{H} \mathbf{x}_{i}\right\|^{2}
$$

subject to

$$
\left\|\left(\mathbf{w}_{i}^{d}\right)^{H} \mathbf{h}_{i i}^{d}\right\|^{2}=1, \quad i=1, \ldots, M .
$$

Define correlation matrix as $\phi_{i}=E\left[\mathbf{x}_{i} \mathbf{x}_{i}^{H}\right]$. The optimal weight vector is given by

$$
\hat{\mathbf{w}}_{i}^{d}=\frac{\phi_{i}^{-1} \mathbf{h}_{i i}^{d}}{\left(\mathbf{h}_{i i}^{d}\right)^{H} \boldsymbol{\phi}_{i}^{-1} \mathbf{h}_{i i}^{d}} .
$$

In traditional power control schemes, the overall transmit powers of all links are minimized, while each link's transmit power is selected so that its SINR is equal to or larger than a fixed and predefined targeted SINR threshold $\gamma_{i}^{d}$ required to maintain the link quality. The power control problem can be defined as

$$
\min _{P_{i}^{d}} \sum_{i=1}^{K} \sum_{d=1}^{D} P_{i}^{d}
$$

subject to

$$
(\mathbf{I}-\mathbf{B F}) \mathbf{P} \geq \mathbf{u}
$$

where $\mathbf{u}=\left[u_{1}^{1}, \ldots, u_{1}^{D}, \ldots, u_{K}^{1}, \ldots, u_{K}^{D}\right]^{T}, \mathbf{P}=\left[P_{1}^{1}, \ldots, P_{1}^{D}, \ldots\right.$, $\left.P_{K}^{1}, \ldots, P_{K}^{D}\right]^{T}, \mathbf{I}$ is the identical matrix, $\mathbf{B}=\operatorname{diag}\left\{\gamma_{1}^{1}, \ldots, \gamma_{1}^{D}\right.$, $\left.\ldots, \gamma_{K}^{1}, \ldots, \gamma_{K}^{D}\right\}$, and

$$
[\mathbf{F}]_{k j}= \begin{cases}0 & \text { if } j=k, \\ \frac{G_{i^{\prime} i}^{d^{\prime}}\left\|\left(\mathbf{w}_{i}^{d}\right){ }^{H} \mathbf{h}_{i^{\prime} i}^{d^{\prime}}\right\|^{2}}{G_{i i}^{d}} & \text { if } j \neq k,\end{cases}
$$

where $i=\lfloor k / D\rfloor, d=\bmod (k, D), i^{\prime}=\lfloor j / D\rfloor, d^{\prime}=\bmod (j, D)$, and $k, j=1 \cdots K D$.

If the spectral radius $\rho(\mathbf{B F})$ [17], that is, the maximum eigenvalue of $B F$, is inside the unit circle, the system has feasible solutions and there exists a positive power allocation vector to achieve the desired targeted SINRs. By PerronFrobenius theorem $[17,18]$, the optimum power vector for this problem is $\hat{\mathbf{P}}=(\mathbf{I}-\mathbf{B F})^{-1} \mathbf{u}$. Many adaptive algorithms $[3,4,19]$ have been developed to reduce the system complexity by the following distributed iteration:

$$
P_{i}^{d}(n+1)=\frac{\gamma_{i}}{G_{i i}^{d}} I_{i}^{d}
$$

where $I_{i}^{d}=\left(\mathbf{w}_{i}^{d}\right)^{H} \mathbf{N}_{i} \mathbf{w}_{i}^{d}+\sum_{(k, j) \neq(i, d)}^{K, D}\left\|\left(\mathbf{w}_{i}^{d}\right)^{H} \mathbf{h}_{k i}^{j}\right\|^{2} P_{k}^{j} G_{k i}^{j}$ and $I_{i}^{d}$ can be easily estimated at the receivers. The power allocation is balanced at the equilibrium when the power update in (11) has converged.

The level of CCI depends on both channel gain and transmit power. The optimal beamforming vector may vary for different powers. Hence the beamforming and power control should be considered jointly. In [4], a joint power control and beamforming scheme has been proposed. An iterative algorithm is developed to jointly update the transmit powers and beamformer weight vectors. The algorithm converges to the jointly optimal transmit power and beamforming solution. The joint iterative algorithm can be summarized by the following two steps:

(i) beamforming in physical layer: MVDR algorithm,

(ii) power update in MAC layer: $\mathbf{P}^{n+1}=\mathbf{B F P}^{n}+\mathbf{u}$,

where power update step can be implemented by using only local interference measurement. But the algorithm assumes the knowledge of SINR and directions of the desired signals or the perfect measurements of channel responses, which are very difficult to get in practice.

\section{JOINT POWER CONTROL AND BLIND BEAMFORMING}

In this section, first we consider how to choose a blind beamforming algorithm that can be used for joint optimization with power control. Then we reformulate the joint power control and blind beamforming problem as a cross layer approach. Finally, an adaptive iterative algorithm is developed.

\subsection{Choosing a blind beamforming algorithm}

The traditional beamforming needs the measurement of spatial responses of the array. A common practice is the use of training sequences [1]. However, it costs bandwidth which 
is very precious and limited in wireless networks. Moreover, the measurement errors can greatly reduce the performance of beamforming. This gives us the motivation to use blind beamforming method to separate and estimate the multiple signals arriving at the antenna array. Since beamforming and power control are two different layer techniques, we need to find the blind beamforming algorithms that allow us to have joint optimization across the layers. In $[13,14]$, a maximum likelihood approach named iterative least squares projection (ILSP) algorithm is proposed. The algorithm explores the finite alphabet property of digital signals. The channel estimation and symbol detection can be implemented at the same time. In addition, a quantity is available for BER performance and can be used for power control optimization [20]. In this subsection, we will briefly review the ILSP algorithm.

Consider the same channel module in (2). The $d$ th mobile inside the $i$ th cell generates binary data $s_{i}^{d}(n)$ with power $P_{i}^{d}$ transmitted over a low delay spread Rayleigh fading channel. The channel and antenna array response is $\mathbf{h}_{i i}^{d}$. The sampled antenna output at the $i$ th base station is given by

$$
\mathbf{x}_{i}(n)=\sum_{d=1}^{D} \mathbf{h}_{i i}^{d} \sqrt{P_{i}^{d} G_{i i}^{d}} s_{i}^{d}(n)+\mathbf{v}_{i}(n),
$$

where $\mathbf{v}_{i}(n)$ includes the $i$ th base station antenna thermal noise and all the CCIs from the other cells, that is,

$$
\mathbf{v}_{i}(n)=\mathbf{n}_{i}(n)+\sum_{\substack{k=1 \\ k \neq i}}^{K} \sum_{d=1}^{D} \mathbf{h}_{k i}^{d} \sqrt{P_{k}^{d} G_{k i}^{d}} s_{k}^{d}(n),
$$

where $\mathbf{n}_{i}(n)$ is the $M \times 1$ sampled thermal noise vector.

The ILSP algorithm works with a shifting window on data blocks of size $N$. Assume that the channel is constant over the $N$ symbol periods. In the $i$ th cell, we obtain the following formulation of the $l$ th data block:

$$
\mathbf{X}_{i}(l)=\mathbf{A}_{i} \mathbf{S}_{i}(l)+\mathbf{V}_{i}(l),
$$

where $l$ is block number,

$$
\begin{aligned}
\mathbf{X}_{i}(l) & =\left[\begin{array}{llll}
\mathbf{x}_{i}(l N+1) & \mathbf{x}_{i}(l N+2) & \cdots & \mathbf{x}_{i}((l+1) N)
\end{array}\right], \\
\mathbf{V}_{i}(l) & =\left[\begin{array}{llll}
\mathbf{v}_{i}(l N+1) & \mathbf{v}_{i}(l N+2) & \cdots & \mathbf{v}_{i}((l+1) N)
\end{array}\right], \\
\mathbf{S}_{i}(l) & =\left[\begin{array}{llll}
\mathbf{s}_{i}(l N+1) & \mathbf{s}_{i}(l N+2) & \cdots & \mathbf{s}_{i}((l+1) N)
\end{array}\right] \\
\mathbf{s}_{i}(n) & =\left[\begin{array}{lll}
s_{i}^{1}(n) & \cdots & s_{i}^{D}(n)
\end{array}\right]^{T}, \\
\mathbf{A}_{i} & =\left[\begin{array}{llll}
\sqrt{P_{i}^{1} G_{i i}^{1}} \mathbf{h}_{i i}^{1} & \cdots & \sqrt{P_{i}^{D} G_{i i}^{D}} \mathbf{h}_{i i}^{D}
\end{array}\right] .
\end{aligned}
$$

We assume that the number of users is known or has been estimated.

The ILSP algorithm uses the finite alphabet property of the input to implement a least squares algorithm that has good convergence properties for the channel with low delay spread. The algorithm is carried out in two steps to alternatively estimate $\mathbf{A}_{i}$ and $\mathbf{S}_{i}$ as follows:

$$
\min _{\mathbf{A}_{i}, \mathbf{S}_{i}} f\left(\mathbf{A}_{i}, \mathbf{S}_{i} ; \mathbf{X}_{i}\right)=\left\|\mathbf{X}_{i}(l)-\mathbf{A}_{i} \mathbf{S}_{i}(l)\right\|^{2}
$$

The first step is a least square minimization problem, where $\mathbf{S}_{i}$ is unstructured and its amplitude is continuous without considering the discrete nature of modulations, while $\mathbf{A}_{i}$ is fixed and equal to the estimated $\hat{\mathbf{A}}_{i}$. In the second step, each element of the solution $\mathbf{S}_{i}$ is projected to its closest discrete values $\hat{\mathbf{S}}_{\mathbf{i}}$. Then a better estimate of $\hat{\mathbf{A}}_{i}$ is obtained by minimizing $f\left(\mathbf{A}_{i}, \hat{\mathbf{S}}_{i} ; \mathbf{X}_{i}\right)$ with respect to $\mathbf{A}_{i}$, keeping $\hat{\mathbf{S}}_{i}$ fixed. We continue this process until estimates of $\hat{\mathbf{A}}_{i}$ and $\hat{\mathbf{S}}_{i}$ are converge. The ILSP algorithm is given in Algorithm 1.

\subsection{Reformulation of joint power control and beamforming}

In traditional joint power control and beamforming, the user's received SINR is larger than or equal to a targeted value to maintain the link quality such as the desired BER. In this paper, we proposed another quantity available from the ILSP algorithm to directly ensure each user's BER. For simplicity, we use BPSK modulation for the analysis and simulation. The other PAM or MQAM modulation methods can be easily extended in a similar way. It has been shown in [14] that the error probability of ILSP algorithm is approximated by

$$
P_{r}\left(s_{i}^{d}\right)=Q\left(\sqrt{\frac{2}{\operatorname{Var}\left[\hat{s}_{i}^{d}(n)\right]}}\right),
$$

where each estimated signal $\hat{s}_{i}^{d}(n)$ has $E\left[\hat{s}_{i}^{d}(n)\right]=s_{i}^{d}(n)$, that is, ILSP is an unbiased estimator with variance

$$
\operatorname{Var}\left[\hat{s}_{i}^{d}(n)\right]=2 \sigma_{i}^{2}\left(\mathbf{A}_{i}^{H} \mathbf{A}_{i}\right)_{d d}^{-1}
$$

where, in our case, $\sigma_{i}^{2}=E\left[\mathbf{v}_{i}(n)^{H} \mathbf{v}_{i}(n)\right]$ and can be estimated by

$$
\sigma_{i}^{2} \approx \frac{1}{N}\left\|\mathbf{X}_{i}-\hat{\mathbf{A}}_{i} \hat{\mathbf{S}}_{i}\right\|^{2}=\frac{1}{N}\left\|\mathbf{V}_{i}\right\|^{2}
$$

In [14], (18) is developed for single cell environment with additive white Gaussian noise. In our case, we need to perform optimization in multicell scenario with CCI. Because there are a large number of CCI sources with similar received powers, by the central limit theorem, we can assume that $\mathbf{v}_{i}(n)$ approaches a zero-mean Gaussian vector. So (18) still holds in our case. From the simulation results in Section 5, we can show that this assumption is valid.

In our proposed joint power control and blind beamforming scheme, the key issue is the quantity $\operatorname{Var}\left[\hat{s}_{i}^{d}(n)\right]$ which is directly related to error performance. $\operatorname{Var}\left[\hat{s}_{i}^{d}(n)\right]$ is a function of $\sigma_{i}^{2}$ and $\mathbf{A}_{i}$, so it is also a function of all $P_{i}^{d}$, for all $i, d$. We want the maximum variance for each user's $\operatorname{Var}\left[\hat{s}_{i}^{d}(n)\right]$ to be less than or equal to a predefined value $\operatorname{var}_{0}$ so that each user's BER is less than the desired value. However, if $\operatorname{var}_{0}$ is too small, each user's transmit power will be too large and cause too much CCI. Under this condition, the system may not be feasible, that is, no matter how large the transmit powers are, the receivers cannot achieve desired BER. So we need a feasibility constraint for $\operatorname{var}_{0}$. The reformulated joint power control and blind beamforming 

(1) Initial $\hat{\mathbf{A}}_{i, 0}$, Step $m=0$.
(2) $m=m+1$
(a) $\overline{\mathbf{S}}_{i, m}=\mathbf{A}_{i, m-1}^{+} \mathbf{X}_{i}$, where $\mathbf{A}_{i, m-1}^{+}=\left(\hat{\mathbf{A}}_{i, m-1}^{H} \hat{\mathbf{A}}_{i, m-1}\right)^{-1} \hat{\mathbf{A}}_{i, m-1}^{H}$,
(b) projection onto finite alphabet
$\hat{\mathbf{S}}_{i, m}=\operatorname{proj}\left[\overline{\mathbf{S}}_{i, m}\right]$,
(c) $\hat{\mathbf{A}}_{i, m}=\mathbf{X}_{i} \hat{\mathbf{S}}_{i, m}^{+}$,
where $\hat{\mathbf{S}}_{i, m}^{+}=\hat{\mathbf{S}}_{i, m}^{H}\left(\hat{\mathbf{S}}_{i, m} \hat{\mathbf{S}}_{i, m}^{H}\right)^{-1}$.
(3) Repeat until $\left(\hat{\mathbf{A}}_{i, m}, \hat{\mathbf{S}}_{i, m}\right) \approx\left(\hat{\mathbf{A}}_{i, m-1}, \hat{\mathbf{S}}_{i, m-1}\right)$.

Algorithm 1

problem is given by

$$
\min _{P_{i}^{d}} \sum_{i=1}^{K} \sum_{d=1}^{D} P_{i}^{d},
$$

subject to

$$
\operatorname{Var}\left(\hat{s}_{i}^{d}(n)\right) \leq \operatorname{var}_{0}, \quad \forall i, d,
$$

where var $_{0}$ is feasible. In order to solve this problem, we need to develop a distributed algorithm such that each user can adapt its transmit power by using only local information. We need to evaluate the feasible range of $\operatorname{var}_{0}$ such that the system is feasible, that is, there exists a possible power allocation vector. The convergence and optimality of the adaptive algorithm will be considered in Section 4 .

\subsection{Adaptive iterative algorithm}

In this subsection, we assume that var $_{0}$ is feasible for the system. We will discuss the feasibility issue in Section 4.1. In ILSP algorithm, the iteration stops when the estimated channel response matrix and symbol matrix have converged. In the algorithm, we use the final channel response matrix $\hat{\mathbf{A}}_{i}$ to substitute $\mathbf{A}_{i}$ in (18). Then the estimation of $\operatorname{Var}\left(\hat{s}_{i}^{d}(n)\right)$ is calculated by

$$
\operatorname{var}_{i}^{d}=2 \sigma_{i}^{2}\left(\hat{\mathbf{A}}_{i}^{H} \hat{\mathbf{A}}_{i}\right)_{d d}^{-1}
$$

In the uplink, the value of $\operatorname{var}_{i}^{d}$ is obtained in the base station and compared with the desired $\operatorname{var}_{0}$. If $\operatorname{var}_{i}^{d}$ is too large, it means that the BER for the $d$ th user is too large and consequently, the $d$ th user's power needs to be increased. If $\operatorname{var}_{i}^{d}$ is too small, it is unnecessary to have such a high power for the $d$ th user. Consequently, the power needs to be reduced. The power update stops when transmit powers have converged in the consecutive iterations, that is, $\operatorname{var}_{i}^{d} \approx \operatorname{var}_{0}$. Each user's power is updated by the simple feedback of $\lambda=\operatorname{var}_{i}^{d} / \operatorname{var}_{0}$ from the base station. The power update scheme can be easily implemented in a distributed manner. In each iteration, the power is updated by

$$
P_{i}^{d}(m+1)=\lambda P_{i}^{d}(m),
$$

where $m$ is the iteration number.
With the above power update equation, we develop the following joint adaptive power control and blind beamforming algorithm. The algorithm is initialized by some feasible power allocation vector $\mathbf{P}(0)$ and some approximate channel estimation $\hat{\mathbf{A}}_{i, 0}$ [13]. The user's BER may be larger than the desired value during the initialization. In each iteration, first, ILSP blind estimate algorithm is applied to estimate the antenna array responses and the transmitted signals. Then $\operatorname{var}_{i}^{d}$ is calculated. The new transmit power is updated by (23). The iteration is stopped by comparing the power vector of the two consecutive iterations. When the algorithm stops, each user's desired BER will be satisfied. The adaptive algorithm is summarized in Algorithm 2.

With the adaptive algorithm, we can construct a joint power control and blind beamforming system as shown in Figure 1. The variance calculator module calculates the estimation $\operatorname{var}_{i}^{d}$ from the ILSP module. The updating information of transmit powers is computed by the power update module. Then the simple power update information is sent back to mobiles via the feedback channels. When the algorithm converges, the output data from the ILSP module will have the desired BER.

\section{ANALYSIS AND CONVERGENCE OF THE ALGORITHM}

\subsection{Convergence analysis}

In this subsection, we analyze the condition for our proposed algorithm to converge, that is, we find the feasible range for $\operatorname{var}_{0}$. Then we prove that the power update converges to a unique solution when the system is feasible, while the blind beamforming may not converge to a unique solution. So our proposed joint power control and blind beamforming algorithm may have local minima because of the inherited characteristics of the blind estimation. We will propose a method to avoid the local minima. From the simulation results in Section 5, we can show that even with the possible local minima, the proposed algorithm performs comparably well with the traditional joint power control and beamforming algorithm.

Consider the transmission from the $d$ th mobile to its associated $i$ th base station with $\mathbf{h}_{i i}^{d}$ and $G_{i i}^{d}$ being the channel response and link gain, respectively, and $\mathbf{A}_{i}$ being the channel response matrix. We want to find the expression $\operatorname{Var}\left[\hat{s}_{i}^{d}(n)\right]$ in (18). Then we will analyze the conditions for the convergence of our algorithm. We have

$$
\left[\mathbf{A}_{i}^{H} \mathbf{A}_{i}\right]_{j k}=\sqrt{P_{i}^{j} P_{i}^{k} G_{i i}^{j} G_{i i}^{k}}\left(\mathbf{h}_{i i}^{j}\right)^{H} \mathbf{h}_{i i}^{k} .
$$

The $\operatorname{det}\left(\mathbf{A}_{i}^{H} \mathbf{A}_{i}\right)$ can be expanded by the following alternating sum form:

$$
\operatorname{det}\left(\mathbf{A}_{i}^{H} \mathbf{A}_{i}\right)=P_{i}^{1} G_{i i}^{1} \cdots P_{i}^{D} G_{i i}^{D} f_{1}\left(\mathbf{h}_{i i}\right) \text {, }
$$

where $\mathbf{h}_{i i}=\left[\mathbf{h}_{i i}^{1}, \ldots, \mathbf{h}_{i i}^{D}\right]$ and $f_{1}\left(\mathbf{h}_{i i}\right)$ is a real function of channel responses $\mathbf{h}_{i i}^{d}$, for all $d$. Then it follows from the cofactor method of matrix inverse [17] that 
(1) Given $\mathbf{P}(0), \operatorname{var}_{0}, m=0$, and $\hat{\mathbf{A}}_{i}=\hat{\mathbf{A}}_{i, 0}$.

(2) Received data block at base station $i$,

(i) ILSP blind estimation to get $\hat{\mathbf{A}}_{i}$;

(ii) For each mobile $d$ inside $i$ th cell, $\operatorname{var}_{i}^{d}=2 \hat{\sigma}_{i}^{2}\left(\hat{\mathbf{A}}_{i}^{H} \hat{\mathbf{A}}_{i}\right)_{d d}^{-1}$

$$
\begin{aligned}
& \lambda=\frac{\operatorname{var}_{i}^{d}}{\operatorname{var}_{0}} \\
& P_{i}^{d}(m+1)=\lambda P_{i}^{d}(m) ;
\end{aligned}
$$

(iii) $\hat{\mathbf{A}}_{i, 0}=\hat{\mathbf{A}}_{i}$.

(3) $m=m+1$. Go to step 2;

Repeat until $\mathbf{P}_{i}(m) \approx \mathbf{P}_{i}(m-1)$, for all $i$.

Algorithm 2: Joint power control and blind beamforming algorithm.

$$
\left(\mathbf{A}_{i}^{H} \mathbf{A}_{i}\right)_{d d}^{-1}=\frac{f_{2}^{d}\left(\mathbf{h}_{i i}\right) \prod_{j=1, j \neq d}^{j=D} P_{i}^{j} G_{i i}^{j}}{f_{1}\left(\mathbf{h}_{i i}\right) \prod_{j=1}^{j=D} P_{i}^{j} G_{i i}^{j}}=\frac{f_{3}\left(\mathbf{h}_{i i}\right)}{P_{i}^{d} G_{i i}^{d}},
$$

where $f_{2}^{d}\left(\mathbf{h}_{i i}\right)$ is a real function of channel responses $\mathbf{h}_{i i}^{j}, j \neq$ $d$, and $f_{3}\left(\mathbf{h}_{i i}\right)=f_{2}^{d}\left(\mathbf{h}_{i i}\right) / f_{1}\left(\mathbf{h}_{i i}\right)$.

Because the channels are not reused in the adjacent cells in most of the communication system, we assume that the CCI plus thermal noise in (13) is Gaussian noise with the variance:

$$
\sigma_{i}^{2}=\sum_{j \neq i}^{K} \sum_{d=1}^{D}\left\|\mathbf{h}_{j i}^{d}\right\|^{2} G_{j i}^{d} P_{j}^{d}+M \sigma^{2} .
$$

Now we can calculate $\operatorname{Var}\left[\hat{s}_{i}^{d}(n)\right]$ as

$$
\operatorname{Var}\left[\hat{s}_{i}^{d}(n)\right]=\frac{2 \sigma_{i}^{2}}{\left(\mathbf{A}_{i}^{H} \mathbf{A}_{i}\right)_{d d}}=\frac{2 \sigma_{i}^{2}}{P_{i}^{d} G_{i i}^{d}} f_{3}\left(\mathbf{h}_{i i}\right) .
$$

An interesting result is that $\operatorname{Var}\left(\hat{s}_{k}^{d}(n)\right)$ is independent of the transmit powers of the other mobiles in the same cell. So the main concern for power control is intercell CCI. Substitute into (23), the power update equation can be expressed as

$$
P_{i}^{d}(n+1)=\frac{\sum_{j \neq i}^{K} \sum_{d=1}^{D}\left\|\mathbf{h}_{j i}^{d}\right\|^{2} G_{j i}^{d} P_{j}^{d}+M \sigma^{2}}{G_{i i}^{d} \operatorname{var}_{0}} f_{3}\left(\mathbf{h}_{i i}\right) .
$$

In matrix form, we define a matrix $\mathbf{Q}$ as

$$
[\mathbf{Q}]_{k j}= \begin{cases}G_{i^{\prime} i}^{d^{\prime}} f_{4}^{k j} / G_{i i}^{d} & \text { if } i^{\prime} \neq i, \\ 0 & \text { otherwise, }\end{cases}
$$

where $i=\lfloor k / D\rfloor, d=\bmod (k, D), i^{\prime}=\lfloor j / D\rfloor, d^{\prime}=\bmod (j$, $D)$, and $f_{4}^{k j}=\left\|\mathbf{h}_{j i}^{d}\right\|^{2} f_{3}\left(\mathbf{h}_{i i}\right)$. The matrix expression of (29) for the whole network can be written as

$$
\mathbf{P}(n+1)=\frac{1}{\operatorname{var}_{0}} \mathbf{Q P}(n)+\mathbf{u}
$$

where $\mathbf{P}=\left[P_{1}^{1} \cdots P_{1}^{D}, \ldots, P_{K}^{1} \cdots P_{K}^{D}\right]^{T}, \mathbf{u}=\left[u_{1}, \ldots, u_{D K}\right]^{T}$, and

$$
u_{j}=\frac{f_{3}\left(\mathbf{h}_{i i}\right) M \sigma^{2}}{G_{i i}^{d} \operatorname{var}_{0}} .
$$

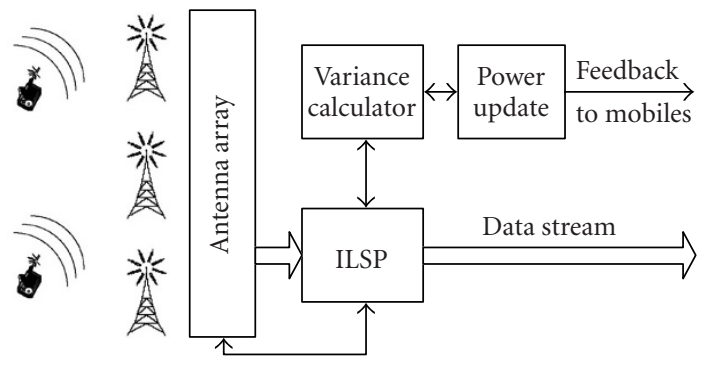

FIGURE 1: Joint power control and blind beamforming system.

By Perron-Frobenius theorem [17], the power update in (31) has the equilibrium

$$
\mathbf{P}=\left(\mathbf{I}-\frac{1}{\operatorname{var}_{0}} \mathbf{Q}\right)^{-1} \mathbf{u} .
$$

If $\left(\mathbf{I}-\left(1 / \operatorname{var}_{0}\right) \mathbf{Q}\right)$ is positive definite, that is, the spectrum radius $|\rho(\mathbf{Q})|<\operatorname{var}_{0}$, the positive power vector exists and the power update converges. Under this condition, the system is converged when $\operatorname{Var}\left[\hat{s}_{i}^{d}(n)\right]=\operatorname{var}_{0}$. From the simulation results in Section 5, we will see that our algorithm converges rapidly to the desired $\operatorname{var}_{0}$ if $|\rho(\mathbf{Q})|<\operatorname{var}_{0}$.

When $\operatorname{var}_{0}$ is too small and less than $\rho(\mathbf{Q})$, the system is not feasible and the adaptive algorithm diverges. In order to prevent the algorithm from diverging, the system will detect the severity of CCI. If the system detect $\rho(\mathbf{Q})$ approaches $\operatorname{var}_{0}$ or the transmit powers increase very fast, $\operatorname{var}_{0}$ will be increased so that users will reduce their transmit powers and CCI will be alleviated.

Following the same proof in [19], we can prove that the power update in (29) converges to a unique solution. Suppose that $\hat{\mathbf{P}}$ and $\mathbf{P}^{*}$ are two different converge power allocation vectors. Without loss of generality, we assume that $\beta=\max _{l}\left(\hat{P}_{l}^{d} / P_{l}^{d *}\right)>1$ such that $\beta \mathbf{P}^{*} \geq \hat{\mathbf{P}}$. We can find an index $i$ such that $\beta P_{i}^{d *}=\hat{P}_{i}^{d}$. We have

$$
\begin{aligned}
\hat{P}_{i}^{d} & =\frac{\sum_{j \neq i}^{K} \sum_{d=1}^{D}\left\|\mathbf{h}_{j i}^{d}\right\|^{2} G_{j i}^{d} \hat{P}_{j}^{d}+M \sigma^{2}}{G_{i i}^{d} \operatorname{var}_{0}} f_{3}\left(\mathbf{h}_{i i}\right) \\
& \leq \frac{\sum_{j \neq i}^{K} \sum_{d=1}^{D}\left\|\mathbf{h}_{j i}^{d}\right\|^{2} G_{j i}^{d} \beta P_{j}^{d *}+M \sigma^{2}}{G_{i i}^{d} \operatorname{var}_{0}} f_{3}\left(\mathbf{h}_{i i}\right) \\
& <\beta \frac{\sum_{j \neq i}^{K} \sum_{d=1}^{D}\left\|\mathbf{h}_{j i}^{d}\right\|^{2} G_{j i}^{d} P_{j}^{d *}+M \sigma^{2}}{G_{i i}^{d} \operatorname{var}_{0}} f_{3}\left(\mathbf{h}_{i i}\right) \\
& =\beta P_{i}^{d *} .
\end{aligned}
$$

The above contradiction implies that the power update equation (23) will converge to a unique solution. However, because the solution of blind beamforming may not be unique [14], our proposed joint scheme may fall into local minima. In order to prevent such local minima, we propose the following scheme to avoid the local minima.

When the two users are not well separated in the angle, that is, the array response $\mathbf{A}_{i}$ is ill-conditioned. The ILSP algorithm can converge to some fixed points that are not the global minima. In this case, instead of projecting 
unstructured continuous estimated symbols to the closest discrete values in ILSP algorithm, we enumerate over all $\Omega^{D}$ possible vectors $S_{i}^{j} \in \Omega^{D}$ and choose the one that minimizes

$$
\hat{\mathbf{S}}_{i}(n)=\underset{\mathbf{S}_{i}^{j} \in \Omega^{D}}{\arg \min }\left\|\mathbf{X}_{i}(n)-\mathbf{A}_{i} \mathbf{S}_{i}^{j}\right\|^{2}, \quad \forall j,
$$

where $\Omega$ is the modulation constellation alphabet. This enumerating method has a better performance but a higher complexity. If the global minimum is still not achieved, it has been shown in [13] that usually one or two reinitializations with random guess are sufficient to yield the global minimum. So we can have two or three parallel structures with different initial values to calculate ILSP algorithm. Then we select the minimal one. The probability of staying in a local minimum will be greatly reduced.

\subsection{Cramer-Rao lower bound}

In our proposed joint power control and blind beamforming system, the performance of each user's BER is determined by the noise variance, channel conditions, and power allocation. When the additive noise is a zero-mean Gaussian random process, the estimation performance of the unbiased estimator is bounded by the CRB. In this subsection, we derive the covariance matrix for the parameters of the thermal noise variance, the input symbols, and the power allocation vector for the CRB. The results will help us analyze the effects of power control on the users' symbol estimation performances in this multicell system.

For simplicity, we assume that the data are modulated as BPSK, that is, $\mathbf{S}(n) \in \Omega^{K D}$, where $\Omega=\{ \pm 1\}$. Similar to the performance analysis of ILSP in [14], we assume that the channel responses are known (the algorithm itself doesn't need such information). The parameters for Fisher information matrix are $\vartheta=\left[\sigma^{2}, \mathbf{S}(1), \ldots, \mathbf{S}(N), \mathbf{P}\right]$. The likelihood function $L$ of the received data $\mathbf{X}(n)$ is given by

$$
\begin{aligned}
L[\mathbf{X}(1) \cdots \mathbf{X}(N)] \\
=\frac{1}{\left(\pi \sigma^{2}\right)^{M K N}} \\
\quad \times \exp \left\{-\frac{1}{\sigma^{2}} \sum_{n=1}^{N}[\mathbf{X}(n)-\mathbf{A S}(n)]^{H}[\mathbf{X}(n)-\mathbf{A S}(n)]\right\} .
\end{aligned}
$$

The Fisher information matrix is calculated by

$$
\begin{aligned}
\mathbf{I}(\theta)_{i j} & =-E\left[\frac{\partial^{2} \ln (L)}{\partial \theta_{i} \partial \theta_{j}}\right] \\
& =\left[\begin{array}{ccccc}
\frac{M K N}{\sigma^{4}} & 0 & \cdots & 0 & 0 \\
0 & \mathbf{Q} & \cdots & 0 & \mathbf{R}(1) \\
\vdots & \vdots & \ddots & \vdots & \vdots \\
0 & 0 & \cdots & \mathbf{Q} & \mathbf{R}(N) \\
0 & \mathbf{R}(1) & \cdots & \mathbf{R}(N) & \mathbf{R}_{P}
\end{array}\right],
\end{aligned}
$$

where $\mathbf{Q}, \mathbf{R}(n)$, and $\mathbf{R}_{P}$ are derived in the appendix.
In order to see the effect of the proposed power control on the symbol estimation errors, we define the average mean square error (AMSE) as a performance measure of the symbol estimation:

$$
\operatorname{AMSE}=\frac{1}{N} \sum_{n=1}^{N} \frac{\|\hat{\mathbf{S}}(n)-\mathbf{S}(n)\|^{2}}{\|\mathbf{S}(n)\|^{2}} .
$$

Because we use BPSK modulation, $\|\mathbf{S}(n)\|^{2}=D K$, for all $n$, and AMSE is the variance bounded by CRB. The CRB for the symbol estimation can be obtained directly from the inverse of Fisher information matrix, that is,

$$
\text { AMSE } \geq \frac{1}{N D K} \sum_{n=1}^{N} \sum_{j=1}^{D K}\left(\mathbf{I}^{-1}(\theta)\right)_{\mathbf{S}^{j}(n) \mathbf{S}^{j}(n)},
$$

where $\mathbf{S}^{j}(n)$ is the $j$ th element of $\mathbf{S}(n)$. How close AMSE is to the CRB will show the relative efficiency of our proposed algorithm.

\section{SIMULATION RESULTS}

A network with 50 cells is simulated as shown in Figure 2. Each hexagonal cell's radius is $1000 \mathrm{~m}$. Two adjacent cells do not share the same channel. In each cell, one base station is placed at the center. Two mobiles are placed randomly with uniform distribution. Each mobile transmits BPSK data over Rayleigh fading channels. Each base station employs fourelements antenna array. The noise level is $\sigma=1$. The transmit frame has $N=1000$ data symbols. Our shaping function is raised cosine function.

Path loss is due to the decay of the intensity of a propagating radio wave. In our simulations, we use the two slope path loss model [21] to obtain the average received power as a function of distance. According to this model, the average path loss is given by

$$
G=\frac{C}{r^{a}\left(1+r \lambda_{c} /\left(4 h_{b} h_{m}\right)\right)^{b}},
$$

where $C$ is a constant, $r$ is the distance between the mobile and the base station, $a$ is the basic path loss exponent (approximately two), $b$ is the additional path loss component (ranging from two to six), $h_{b}$ is the base station antenna height, $h_{m}$ is the mobile antenna height, and $\lambda_{c}$ is the wavelength of the carrier frequency. We assume the mobile antenna height is $2 \mathrm{~m}$ and the base station antenna height is $50 \mathrm{~m}$. The carrier frequency is $900 \mathrm{MHz}$.

In Figure 3, we show the analytical and numerical performance of ILSP, compared with MVDR with perfect channel estimation. The numerical results with CCI match the analytical results well especially at high SINR range, which proves our assumption that $\mathbf{V}_{i}(n)$ can be treated as Gaussian noise when the number of CCIs is large. Our proposed joint power control and blind beamforming has only about $1-2 \mathrm{~dB}$ performance loss over traditional power control and MVDR beamforming with perfect channel estimation. However, MVDR beamforming needs additional 


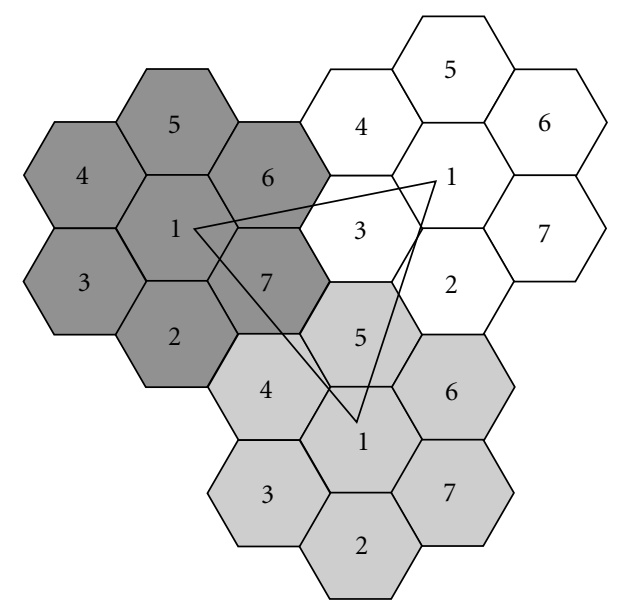

Figure 2: Simulation setup.

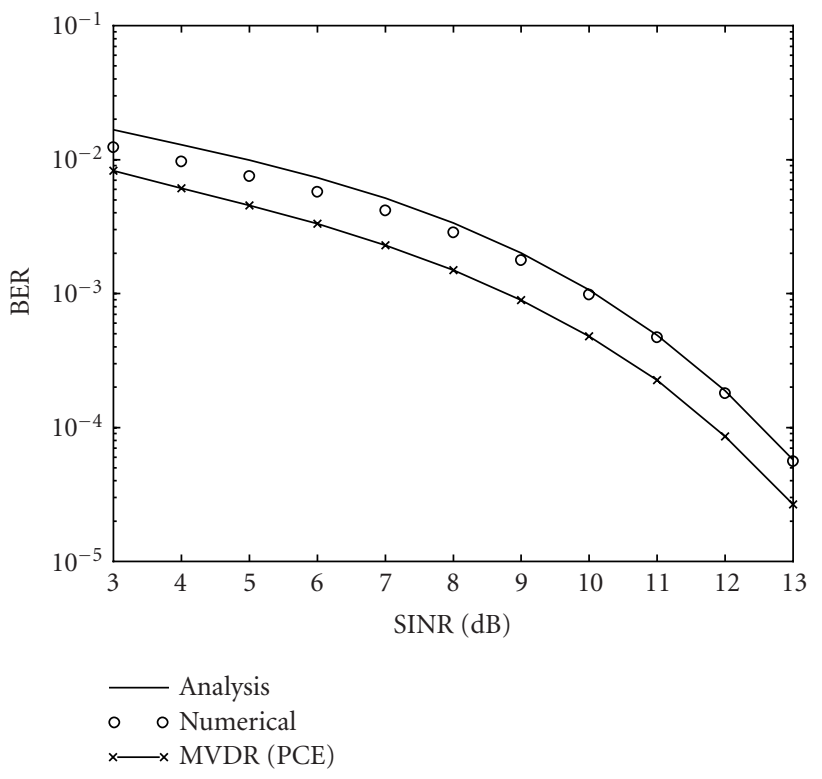

FIGURE 3: ILSP performance.

training sequence to estimate the channel and SINR with prior information that may not be available in practice.

In reality, perfect channel estimation is hard to obtain. In Figure 4, we show the effect of directions of arrivals (DOA) estimation error on the traditional joint power control and MVDR beamforming and our algorithm. In Figure $4 \mathrm{a}$, we compare the BER performance while the transmit power allocation is the same for both algorithms. We can see from the curves that when the channel estimation error for DOA is greater than about 2 degrees, the blind beamforming algorithm outperforms the traditional MVDR. In Figure $4 \mathrm{~b}$, we compare the overall transmit power while BER performance is the same for both algorithms. We can see that the blind beamforming algorithm needs a little bit more transmit powers when the DOA estimation error is small. However, the

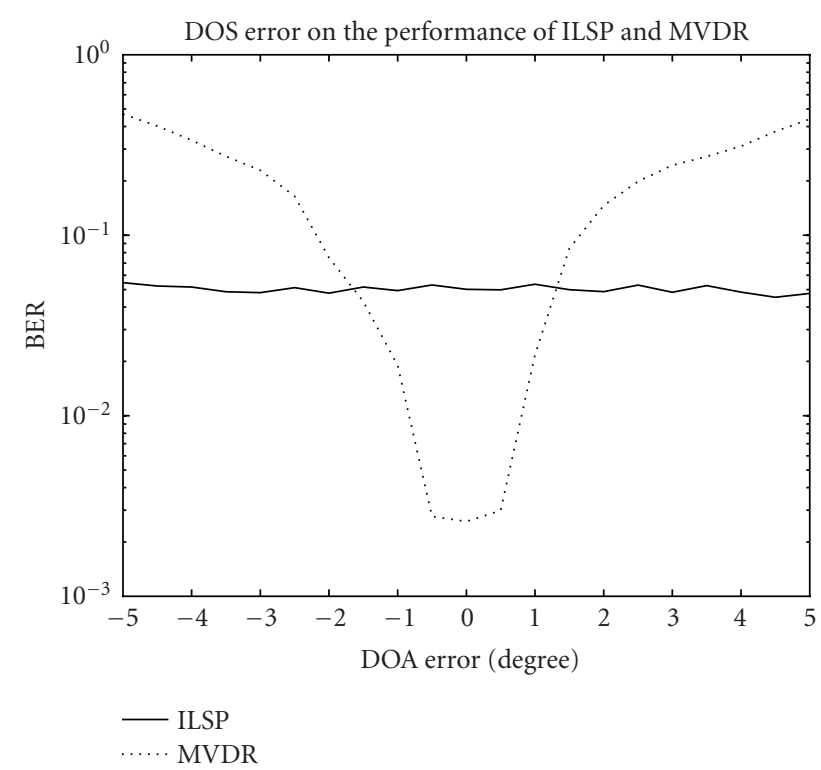

(a) BER.

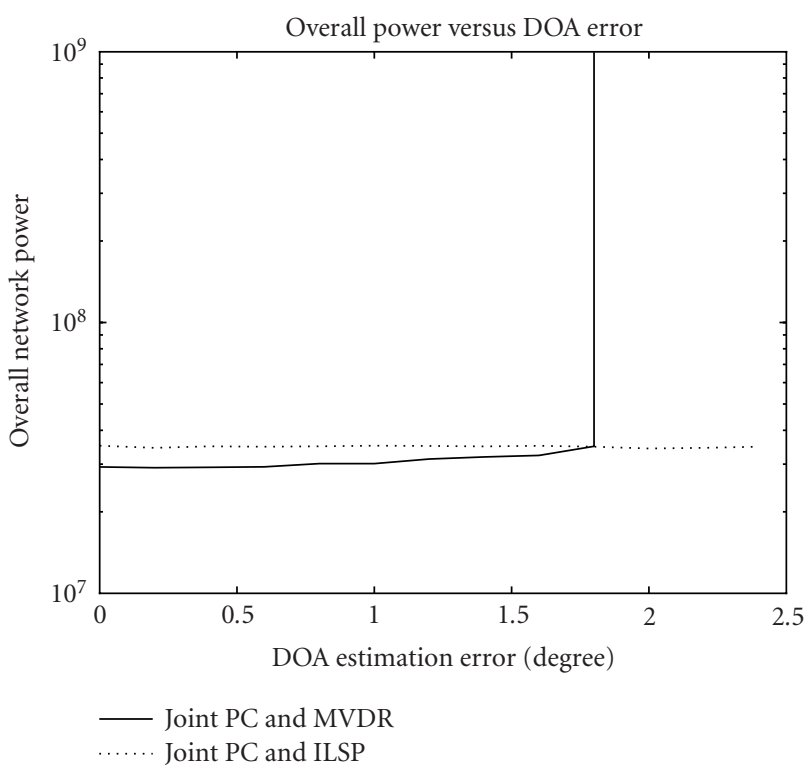

(b) Overall power.

Figure 4: Effects of DOA estimation error.

traditional power control with MVDR method will diverge when the DOA estimation error is about 2 degrees. Our proposed joint power control and beamforming algorithm will always converge regardless the DOA variations. When the mobiles are moving, DOA are changing and this will cause the channel estimation errors. The traditional MVDR beamformer may not be aware of the changing and still use the obsolete $\mathbf{h}_{i i}^{d}$ in (7). This will greatly increase BER and transmit powers of the joint power control and MVDR method. The proposed blind scheme will automatically track and adapt to the changes and so it is more robust to channel estimation 
errors. Consequently, our algorithm is more robust in applications where usually only the inaccurate channel and SINR estimations are available. It is worthy to mention that the proposed scheme is more sensitive to fast channel varying and the complexity is much higher compared to the traditional training sequence-based algorithm. However, our scheme saves the transmission bandwidth by eliminating the training sequences and is more robust to channel estimation errors.

In Figure 5, we show the numerical results of BER and the overall transmit power versus $\operatorname{var}_{0}$ for the proposed joint blind beamforming and power control algorithm. When var 0 is decreasing from a large number, BER decreases and the overall power increases slightly. Within a reasonable BER range such as $\mathrm{BER}=10^{-3}$ to $\mathrm{BER}=10^{-5}$, we can calculate the threshold of $\operatorname{var}_{0}$ for the desired BER. After $\operatorname{var}_{0}$ decreases to a specific value, the overall transmit power increases and BER decreases quickly. This is because the CCI is too large and $\operatorname{var}_{0} \rightarrow \rho(\mathbf{Q})$. After $\operatorname{var}_{0}$ is smaller than some value, the algorithm diverges. Consequently, there is no feasible power control solution, that is, no matter how large the transmit powers are, the receivers cannot ensure the desired BER. This proves that our algorithm behaves exactly the same as the traditional power control algorithm, except that our algorithm directly ensures BER instead of each user's SINR. There is a trade-off between the overall transmit power and BER, while $\operatorname{var}_{0}$ is the bridge between the two quantities.

In Figure 6, we show the distribution of the number of iterations required for the convergence of our proposed algorithm with different values of var $_{0}$. The convergence criteria is that the maximum difference of users' transmit powers between two consecutive iterations is less than 3\%. When $\operatorname{var}_{0}$ is within the range that the system is feasible, we can see that our algorithm converges within a small number of iterations, which demonstrates that our algorithm is robust in the wireless communication systems if the channel gains and topologies have been changed. When var $_{0}$ is large, that is, the desired BER is large, the algorithm converges slower. This is because the transmit powers are small when $\operatorname{var}_{0}$ is large. Consequently, the $\operatorname{var}_{i}^{d}$ estimation is poor and more iterations are needed for the convergence.

In Figure 7, we compare the AMSE and CRB versus var ${ }_{0}$. When $\operatorname{var}_{0}$ is large and the transmit powers of users are small, the CCI is small. The performance of ILSP is close to CRB. The difference is because discrete alphabets are used for transmitted symbols, while there is no such assumption for CRB. When $\operatorname{var}_{0}$ is decreasing, the CCI and our algorithm's AMSE are decreasing because of the increasing transmit powers. In this situation, the CRB is much lower than our algorithm performance. This is because we assume that all the channel conditions including $A_{i j}, i \neq j$, are known for $\mathrm{CRB}$, while our algorithm only estimates $A_{i i}$ and treats transmitted signals from other cells as noise. If an algorithm can take consideration of all $A_{i j}$, for all $i, j$, its performance will be much better and closer to CRB; however, the complexity will be unacceptably high. When $\operatorname{var}_{0}$ is smaller than some value, our algorithm diverges. The transmit powers also di-

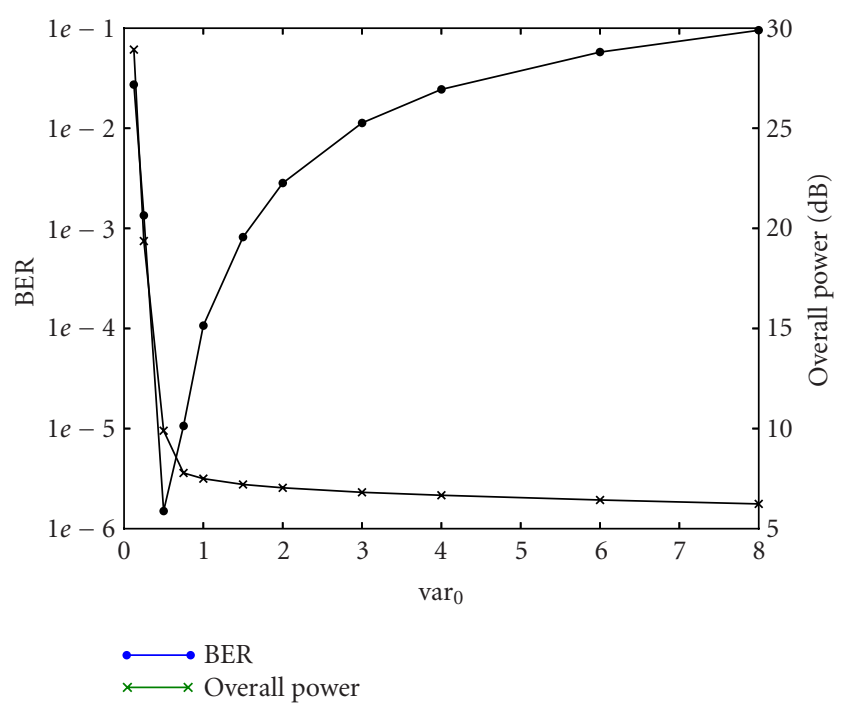

FIgURE 5: BER and the overall power versus $\operatorname{var}_{0}$.

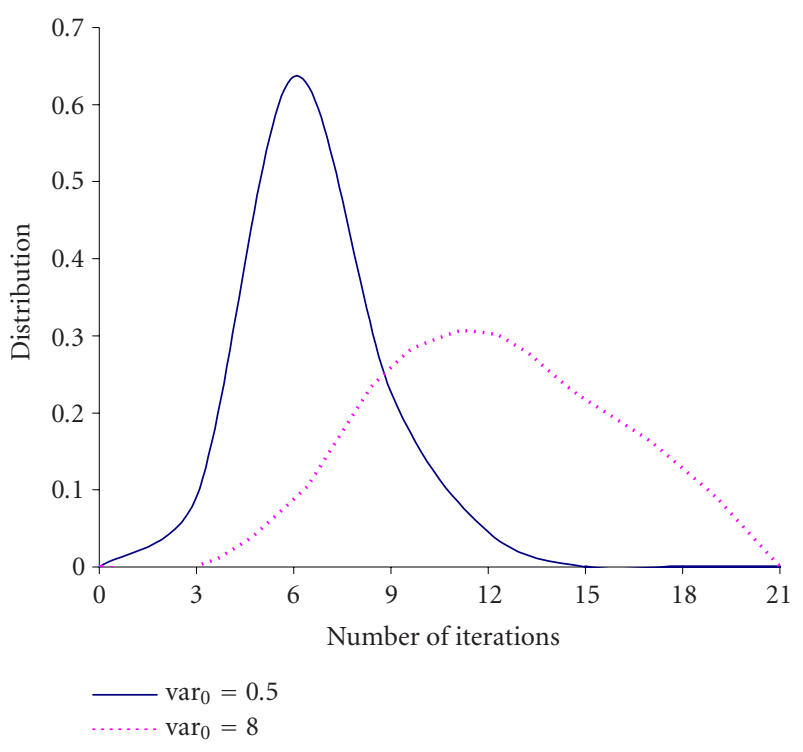

Figure 6: Convergence of the algorithm.

verge to arbitrary large values. But the CRB goes extremely low because SINR can be very high, if we know all the channel responses.

\section{CONCLUSION}

We have proposed a novel joint power control and blind beamforming algorithm that reformulates the power control problem in terms of a quantity directly related to the error performance of the estimation. First, this approach optimizes BER instead of a theoretically indirect SINR. Secondly, the algorithm does not require additional measurements of 


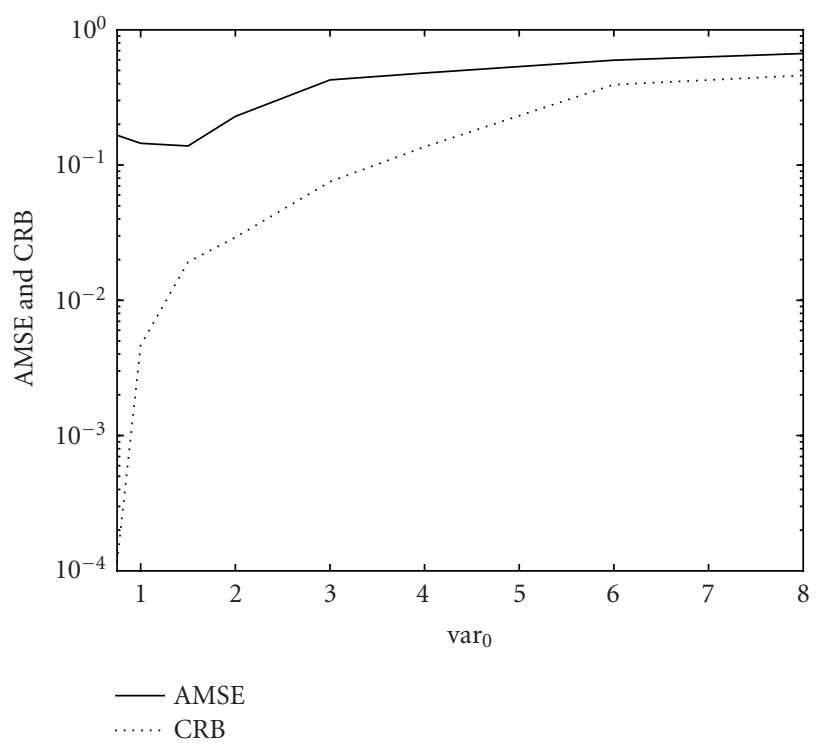

Figure 7: AMSE and CRB versus $\operatorname{var}_{0}$.

channel or SINR, which saves valuable limited bandwidth. Third, our scheme can be easily implemented in a distributed manner. Fourth, our scheme is more robust to channel estimation error. The proof of convergence of the algorithm is derived and supported by simulation results. Performance results show that our algorithm performs well in the situations where the radio spectrum is limited or the good estimations are hard to obtain.

\section{APPENDIX}

From (36), the log likelihood function is

$$
\begin{aligned}
\ln (L)= & -M K N \ln (\pi)-M K N \ln \left(\sigma^{2}\right) \\
& -\frac{1}{\sigma^{2}} \sum_{n=1}^{N}\left[\mathbf{X}^{H}(n)-\mathbf{S}^{T}(n) \mathbf{A}^{H}\right][\mathbf{X}(n)-\mathbf{A S}(n)] .
\end{aligned}
$$

We take partial derivatives of (A.1) with respect to $\sigma^{2}$, $\mathbf{S}(n)$, and $\mathbf{P}$ :

$$
\begin{gathered}
\frac{\partial \ln (L)}{\partial \sigma^{2}}=-\frac{M K N}{\sigma^{2}}+\frac{1}{\sigma^{4}} \sum_{n=1}^{N} \mathbf{e}(n)^{H} \mathbf{e}(n), \\
\frac{\partial \ln (L)}{\partial \mathbf{S}(n)}=\frac{2}{\sigma^{2}} \operatorname{Re}\left\{\mathbf{A}^{H} \mathbf{e}(n)\right\}, \\
\frac{\partial \ln (L)}{\partial P_{i}^{d}}=\frac{2}{\sigma^{2}} \sum_{n=1}^{N} \operatorname{Re}\left\{\mathbf{S}^{T}(n) \frac{d \mathbf{A}^{H}}{d P_{i}^{d}} \mathbf{e}(n)\right\}, \\
\frac{\partial \ln (L)}{\partial \mathbf{P}}=\frac{1}{\sigma^{2}} \sum_{n=1}^{N} \operatorname{Re}\left\{\operatorname{diag}\left(\mathbf{S}^{T}(n)\right) \operatorname{diag}\left(\frac{1}{\mathbf{P}}\right) \mathbf{A}^{H} \mathbf{e}(n)\right\},
\end{gathered}
$$

where $\mathbf{e}(t)=\mathbf{X}(t)-\mathbf{A S}(t)$, and $\operatorname{diag}(1 / \mathbf{P})=\operatorname{diag}\left(1 / P_{1}^{1}, \ldots\right.$, $\left.1 / P_{1}^{D}, \ldots, 1 / P_{K}^{D}\right)$. Using the several results that are proven in $[16,22]$, we have

$$
\begin{gathered}
E\left[\left(\frac{\partial \ln (L)}{\partial \sigma^{2}}\right)^{2}\right]=\frac{M K N}{\sigma^{4}} \\
E\left[\left(\frac{\partial \ln (L)}{\partial \sigma^{2}}\right)\left(\frac{\partial \ln (L)}{\partial \mathbf{S}(n)}\right)^{T}\right]=E\left[\left(\frac{\partial \ln (L)}{\partial \sigma^{2}}\right)\left(\frac{\partial \ln (L)}{\partial \mathbf{P}}\right)^{T}\right]=0, \\
\mathbf{Q}=E\left[\left(\frac{\partial \ln (L)}{\partial \mathbf{S}(n)}\right)\left(\frac{\partial \ln (L)}{\partial \mathbf{S}(r)}\right)^{T}\right]=\frac{2}{\sigma^{2}} \operatorname{Re}\left\{\mathbf{A}^{H} \mathbf{A}\right\} \delta_{n, r}, \\
\mathbf{R}_{P}=E\left[\left(\frac{\partial \ln (L)}{\partial \mathbf{P}}\right)^{2}\right] \\
=\frac{1}{2 \sigma^{2}} \sum_{n=1}^{N} \operatorname{Re}\left\{\operatorname{diag}\left(\mathbf{S}^{T}(n)\right) \operatorname{diag}\left(\frac{1}{\mathbf{P}}\right) \mathbf{A}^{H}\right. \\
\mathbf{R}(n)=E\left[\left(\frac{\partial \ln (L)}{\partial \mathbf{S}(n)}\right)\left(\frac{\partial \ln (L)}{\partial \mathbf{P}}\right)^{T}\right] \\
=\frac{1}{\sigma^{2}} \operatorname{Re}\left\{\mathbf{A} H \mathbf{A} \operatorname{diag}\left(\frac{1}{\mathbf{P}}\right) \operatorname{diag}(\mathbf{S}(n))\right\} .
\end{gathered}
$$

\section{REFERENCES}

[1] S. Haykin, Adaptive Filter Theory, Prentice Hall, Englewood Cliffs, NJ, USA, 3rd edition, 1996.

[2] S. A. Grandhi, R. Vijayan, D. J. Goodman, and J. Zander, "Centralized power control in cellular radio systems," IEEE Trans. Vehicular Technology, vol. 42, no. 4, pp. 466-468, 1993.

[3] R. D. Yates, "A framework for uplink power control in cellular radio systems," IEEE Journal on Selected Areas in Communications, vol. 13, no. 7, pp. 1341-1347, 1995.

[4] F. Rashid-Farrokhi, L. Tassiulas, and K. J. R. Liu, "Joint optimal power control and beamforming in wireless networks using antenna arrays," IEEE Trans. Communications, vol. 46, no. 10 , pp. 1313-1324, 1998.

[5] F. Rashid-Farrokhi, K. J. R. Liu, and L. Tassiulas, "Transmit beamforming and power control for cellular wireless systems," IEEE Journal on Selected Areas in Communications, vol. 16, no. 8, pp. 1437-1450, 1998.

[6] A. Mercado and K. J. R. Liu, "Adaptive QoS for wireless multimedia networks using power control and smart antennas," IEEE Trans. Vehicular Technology, vol. 51, no. 5, pp. 1223$1233,2002$.

[7] Y. C. Liang, F. P. S. Chin, and K. J. R. Liu, "Downlink beamforming for DS-CDMA mobile radio with multimedia services," IEEE Trans. Communications, vol. 49, no. 7, pp. 12881298, 2001.

[8] A. Yener, R. D. Yates, and S. Ulukus, "Interference management for CDMA systems through power control, multiuser detection, and beamforming," IEEE Trans. Communications, vol. 49, no. 7, pp. 1227-1239, 2001.

[9] L. Tong, G. Xu, B. Hassibi, and T. Kailath, "Blind channel identification based on second-order statistics: a frequencydomain approach," IEEE Transactions on Information Theory, vol. 41, no. 1, pp. 329-334, 1995. 
[10] B. Sampath, K. J. R. Liu, and Y. (G.) Li, "Deterministic blind subspace MIMO equalization," EURASIP Journal on Applied Signal Processing, vol. 2002, no. 5, pp. 538-551, 2002.

[11] T. Nguyen and Z. Ding, "Blind CMA beamforming for narrowband signals with multipath arrivals," International Journal of Adaptive Control and Signal Processing, vol. 12, pp. 157$172,1998$.

[12] Y. Li and K. J. R. Liu, "Adaptive blind source separation and equalization for multiple-input/multiple-output systems," IEEE Transactions on Information Theory, vol. 44, no. 7, pp. 2864-2876, 1998.

[13] S. Talwar, M. Viberg, and A. Paulraj, "Blind separation of synchronous co-channel digital signals using an antenna array. I. algorithms," IEEE Transactions on Signal Processing, vol. 44, no. 5, pp. 1184-1197, 1996.

[14] S. Talwar and A. Paulraj, "Blind separation of synchronous cochannel digital signals using an antenna array. II. performance analysis," IEEE Transactions on Signal Processing, vol. 45, no. 3, pp. 706-718, 1997.

[15] Z. Ding and Y. (G.) Li, Blind Equalization and Identification, vol. 9 of Signal processing and communications, Marcel Dekker, New York, NY, USA, 2001.

[16] H. Liu and G. Xu, "Closed-form blind symbol estimation in digital communications," IEEE Transactions on Signal Processing, vol. 43, no. 11, pp. 2714-2723, 1995.

[17] G. H. Golub and C. F. Van Loan, Matrix Computations, Johns Hopkins University Press, Baltimore, Md, USA, 1996.

[18] A. Mercado and K. J. R. Liu, "NP-hardness of the stable matrix in unit interval family problem in discrete time," Systems and Control Letters, vol. 42, no. 4, pp. 261-265, 2001.

[19] G. J. Foschini and Z. Miljanic, "A simple distributed autonomous power control algorithm and its convergence," IEEE Transactions on Vehicular Technology, vol. 42, no. 4, pp. 641-646, 1993.

[20] K. Vissa, F. Rashid-Farrokhi, and K. J. R. Liu, "Combining blind equalization and power control in wireless networks," in Proc. 32nd Asilomar Conference on Signals Systems, and Computers, vol. 1, pp. 88-91, Systems, and Computers, Pacific Grove, 1998 (invited paper).

[21] P. Harley, "Short distance attenuation measurements at $900 \mathrm{MHz}$ and $1.8 \mathrm{GHz}$ using low antenna heights for microcells," IEEE Journal on Selected Areas in Communications, vol. 7, no. 1, pp. 5-11, 1989.

[22] W. M. Steedly and R. L. Moses, "The Cramer-Rao bound for pole and amplitude coefficient estimates of damped exponential signals in noise," IEEE Transactions on Signal Processing, vol. 41, no. 3, pp. 1305-1318, 1993.

Zhu Han received the B.S. degree in electronic engineering from Tsinghua University in 1997, and received the M.S. degree in electrical engineering from the University of Maryland, College Park, in 1999. From 1997 to 2000, he was a Graduate Research Assistant. From 2000 to 2002, he was an Engineer in R\&D group of ACTERNA. In November 2003, he got his Ph.D. degree from the University of Maryland, College Park, where he

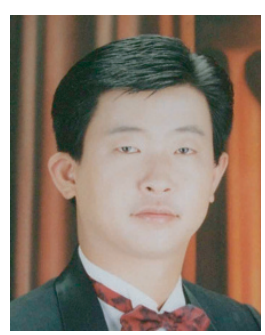
is now a Research Associate. His research interests are wireless resource allocation, game theory approach, OFDM, CDMA, multimedia over wireless, blind estimation, and bioimage processing.
Farrokh R. Farrokhi received the B.S. and M.S. degrees (highest honors) in electrical engineering from Sharif University of Technology, Tehran, Iran, in 1988 and 1992, respectively, and the Ph.D. degree in electrical engineering from the University of Maryland at College Park in 1997. From 1998 to 2000 , he was a member of the technical staff in the Wireless Research Department, Bell Labs, Lucent Technologies. From

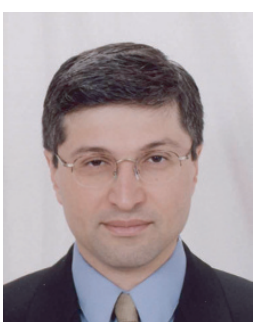
2000 to 2002, he was with the Advanced Research and Development Department, Centillium Communications. Since 2002, he has been with DSP Department, Cognio Inc. His research interests include array and statistical signal processing, wireless communications and networking. Dr. Farrokhi received the 19961997 George Harhalakis Outstanding Systems Engineering Graduate Student Award in recognition of outstanding contributions in cross-disciplinary research from the University of Maryland at College Park.

K. J. Ray Liu received the B.S. degree from the National Taiwan University in 1983, and the Ph.D. degree from UCLA in 1990, both in electrical engineering. He is a Professor of Electrical and Computer Engineering Department and Institute for Systems Research of University of Maryland, College Park. His research contributions encompass broad aspects of multimedia communications and signal processing; wireless communications and networking; information security; signal processing algorithms and architectures; and bioinformatics, in which he has published over 300 refereed papers. Dr. Liu is the recipient of numerous honors and awards including the IEEE Signal Processing Society 2004 Distinguished Lecturer, the 1994 National Science Foundation Young Investigator Award, the IEEE Signal Processing Society's 1993 Senior Award (Best Paper Award), IEEE 50th Vehicular Technology Conference Best Paper Award, Amsterdam, 1999. He also received the George Corcoran Award in 1994 for outstanding contributions to electrical engineering education and the Outstanding Systems Engineering Faculty Award in 1996 in recognition of outstanding contributions in interdisciplinary research, both from the University of Maryland. Dr. Liu is a Fellow of IEEE. Dr. Liu is the Editor-in-Chief of IEEE Signal Processing Magazine and was the Founding Editor-in-Chief of EURASIP Journal on Applied Signal Processing. Dr. Liu is a Board of Governor and has served as Chairman of Multimedia Signal Processing Technical Committee of IEEE Signal Processing Society. 